Acta Histochem. Cytochem. 45 (1): 57-64 (2012)

\title{
Immunohistochemical Mapping of TRK-Fused Gene Products in the Rat Brainstem
}

Shigeko Takeuchi, Chiaki Masuda, Hisae Maebayashi and Ikuo Tooyama

The authors would like to correct an error in the affiliation.

Affiliation 2: "Department of Psychology, Kyoto Prefectural University of Medicine, 465 Kajii-cho, Kamigyo-ku, Kyoto 602-8566, Japan” should read "Department of Psychiatry, Graduate School of Medical Science, Kyoto Prefectural University of Medicine, 465 Kajii-cho, Kamigyo-ku, Kyoto 602-8566, Japan” 